Case Report

\title{
A Rare Case of Complete Stent Fracture, Coronary Arterial Transection, and Pseudoaneurysm Formation Induced by Repeated Stenting
}

\author{
Fumiaki Nakao, ${ }^{1}$ Masashi Kanemoto, ${ }^{1}$ Jutaro Yamada, ${ }^{2}$ Kazuhiro Suzuki, ${ }^{3}$ \\ Hidetoshi Tsuboi, ${ }^{3}$ and Takashi Fujiii ${ }^{1}$ \\ ${ }^{1}$ Department of Cardiology, Yamaguchi Grand Medical Center, 77 Ohsaki, Hofu, Yamaguchi 747-8511, Japan \\ ${ }^{2}$ Division of Cardiology, Department of Medicine and Clinical Science, Yamaguchi University Graduate School of Medicine, \\ 1-1-1 Minami-kogushi, Ube, Yamaguchi 755-8505, Japan \\ ${ }^{3}$ Department of Cardiovascular Surgery, Yamaguchi Grand Medical Center, 77 Ohsaki, Hofu, Yamaguchi 747-8511, Japan
}

Correspondence should be addressed to Fumiaki Nakao; nakao-ymghp@umin.ac.jp

Received 29 June 2015; Revised 12 September 2015; Accepted 28 September 2015

Academic Editor: Mohammad R. Movahed

Copyright (C) 2015 Fumiaki Nakao et al. This is an open access article distributed under the Creative Commons Attribution License, which permits unrestricted use, distribution, and reproduction in any medium, provided the original work is properly cited.

This report describes a rare asymptomatic case of complete stent fracture, coronary arterial transection, and pseudoaneurysm formation in response to repeated stenting. The proximal and distal ends of transected coronary artery were closed, and distal bypass was performed. Coronary arterial transection can occur in patients with repeated stenting as a long-term adverse event.

\section{Introduction}

Stent fracture after drug-eluting stent (DES) deployment is an important issue, because it is strongly associated with restenosis, target legion revascularization, and stent thrombosis [1]. A report of autopsy cases with DES deployment showed stent fracture in $29 \%$ of lesions and restenosis or stent thrombosis in $67 \%$ of cases with gapped stent fracture [2].

Stent fracture can also lead to coronary pseudoaneurysm formation, which can be life-threatening [3]. The incidence of coronary pseudoaneurysm formation after DES deployment is $0.3-4.5 \%$ [4]. Management strategies for coronary pseudoaneurysm include observation, surgical treatment and interventional treatment, such as coil embolization and deployment of a polytetrafluoroethylene- (PTFE-) covered stent [3-5].

\section{Case Report}

A 61-year-old male undergoing chronic hemodialysis had previously underwent rotational atherectomy and stenting (TAXUS Liberte, Boston Scientific Co.) for a long, severely calcified lesion of the right coronary artery (RCA) (first percutaneous coronary intervention [PCI\#1], Figure 1). Six months later, the patient underwent emergent restenting (Cypher, Cordis) for probable stent thrombosis of the midRCA with ST elevation (second PCI [PCI\#2], Figure 2). Four months later, he underwent emergent repeat stenting (Xience V, Abbott Vascular) for probable stent thrombosis of the mid-RCA with ST elevation (third PCI [PCI\#3], Figure 3). Two months later, he was admitted for followup coronary angiography (CAG) and was noted to be asymptomatic. CAG showed pseudoaneurysm formation in the mid-RCA (see Figures 4(a), 4(b), and 4(c) and see Clip 1 in Supplementary Material available online at http://dx.doi.org/10.1155/2015/192853), and X-ray fluorography showed complete stent fracture (Figure 4(d)). Coronary transection was suspected, because of findings of complete stent fracture and contrast media oozing all around the part of stent fracture.

\section{Discussion}

Risk factors for stent fracture include RCA stenting, long stenting, overlapped stenting, and stenting on a hinge point $[6,7]$. The present patient underwent long and overlapped 


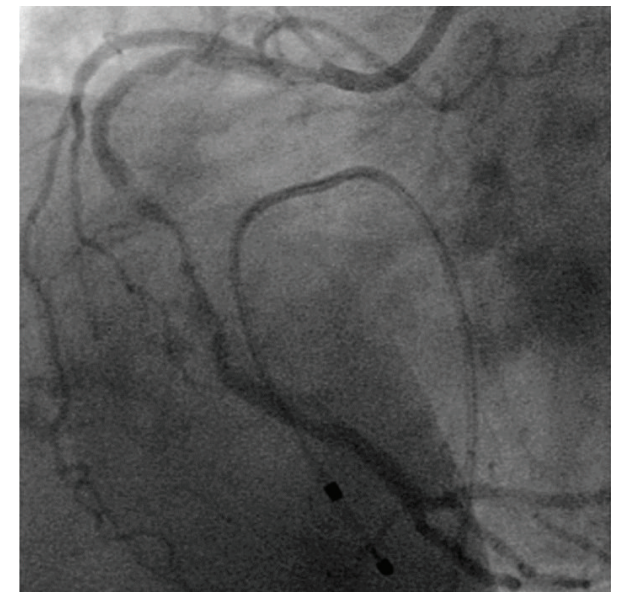

(a)

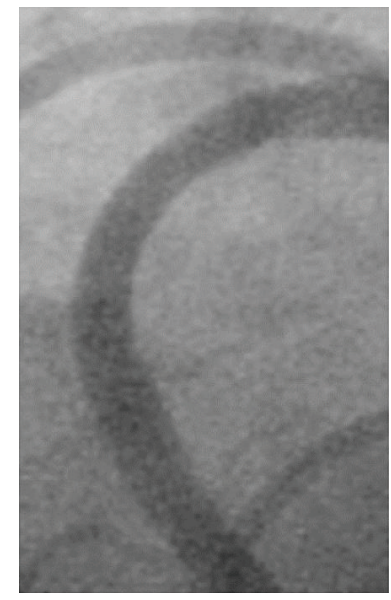

(b)

FIGURE 1: First percutaneous coronary intervention (PCI\#1). (a) Baseline coronary angiography (CAG). (b) CAG after first stenting.

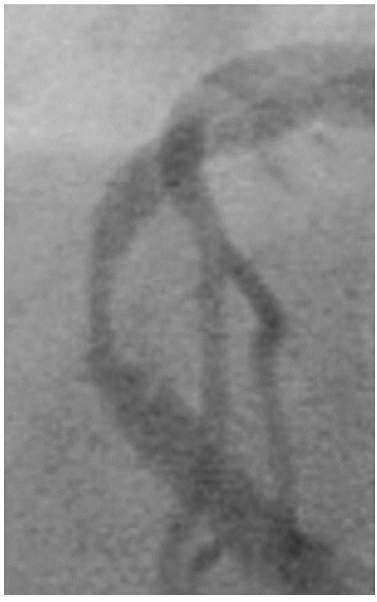

(a)

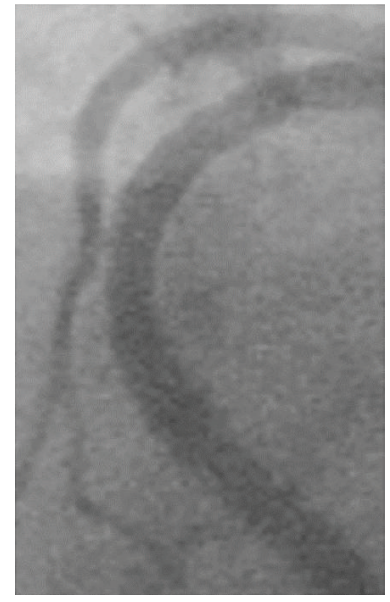

(b)

FIGURE 2: Second percutaneous coronary intervention (PCI\#2). (a) Baseline coronary angiography (CAG). (b) CAG after second stenting.

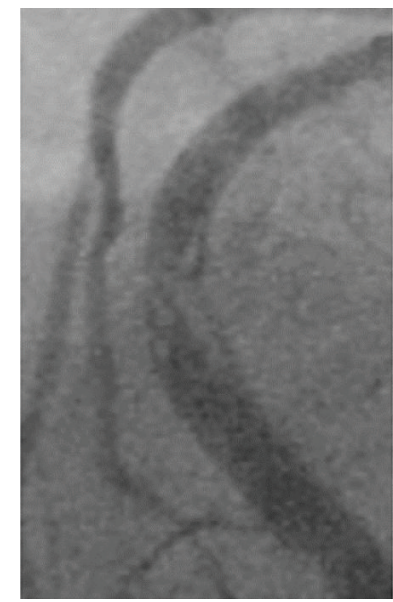

(a)

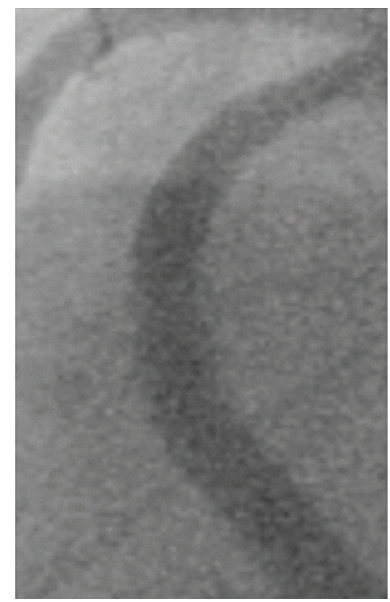

(b)

FIgURE 3: Third percutaneous coronary intervention (PCI\#3). (a) Baseline coronary angiography (CAG). (b) CAG after third stenting. 


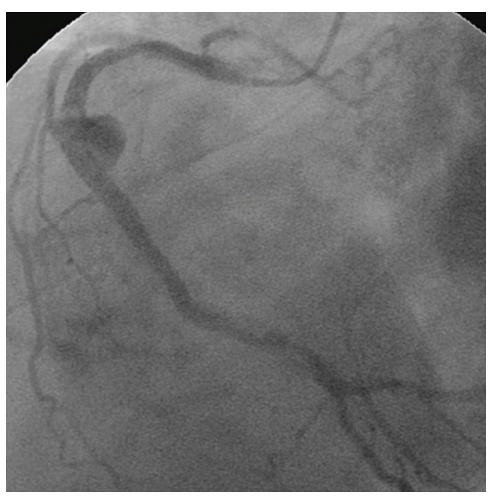

(a)

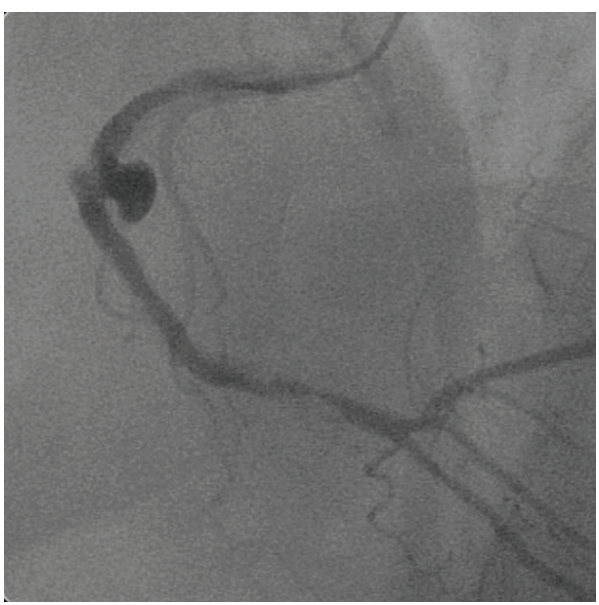

(c)

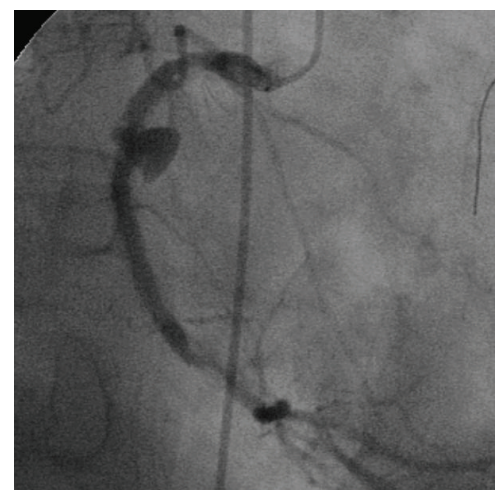

(b)

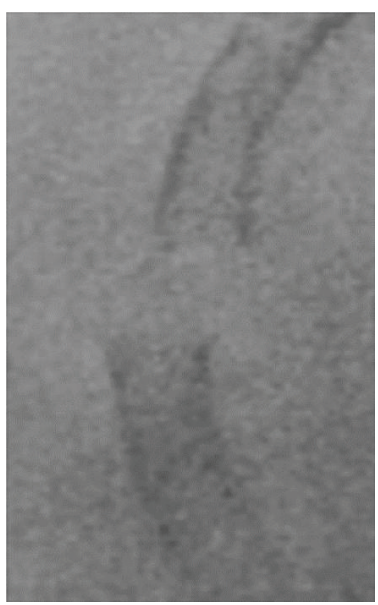

(d)

FIGURE 4: Follow-up coronary angiography showing pseudoaneurysm formation. Left anterior oblique (LAO) view (a), right anterior oblique view (b), and LAO-cranial view (c). (d) X-ray fluorography showing complete stent fracture.

stenting within the RCA and therefore was at high risk for stent fracture. Drugs and polymers of DES may induce vascular inflammation and delay vascular healing [8], and they also can contribute to pseudoaneurysm formation. In this case, the vessel wall was likely exposed to a relatively high dose of DES drug and polymer (due to three overlapping stents).

Surgical treatment and a PTFE-covered stent deployment were considered for this case. However, a guidewire could perforate the wall of the pseudoaneurysm, and deployment of the PTFE-covered stent might be difficult, because previous procedures required the mother and child (4 in 6) technique. If repeated stenting for stent fracture was performed, stent fracture might occur repeatedly, leading to lethal stent thrombosis or blow-out rupture of the pseudoaneurysm. Therefore, surgical management was selected for this case. During surgery, the pseudoaneurysm was visualized in the visceral adipose tissue (arrow heads, Figure 5(a)). After the pseudoaneurysm was opened (Figure 5(b)), coronary transection was confirmed (arrows, Figure 5(b)). The proximal and distal transected ends of the mid-RCA could not be ligated because of protrusion of the overlapped fractured struts (arrows, Figure 5(c)). Therefore, proximal and distal transected ends of the mid-RCA were closed (Figure 5(d)), and distal bypass was performed.

In conclusion, this case report described a rare asymptomatic case of complete stent fracture, coronary arterial transection, and pseudoaneurysm formation in response to repeated stenting. Coronary arterial transection can occur in patients with repeated stenting as a long-term adverse event.

\section{Conflict of Interests}

The authors declare that there is no conflict of interests with regard to this report.

\section{Acknowledgments}

The authors thank Tooru Ueda, Takamasa Oda, and Yasuhiro Ikeda of the Department of Cardiology, Ymaguchi Grand Medical Center, for their support and thank Takayuki Okamura and Masafumi Yano of the Division of Cardiology, Department of Medicine and Clinical Science, Yamaguchi 


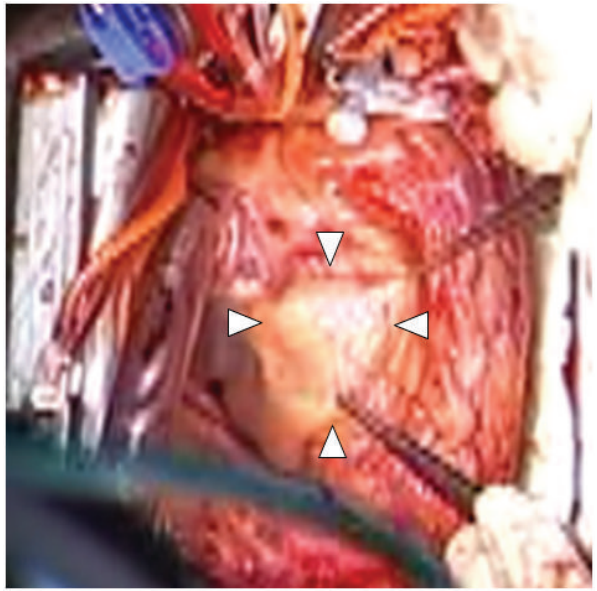

(a)

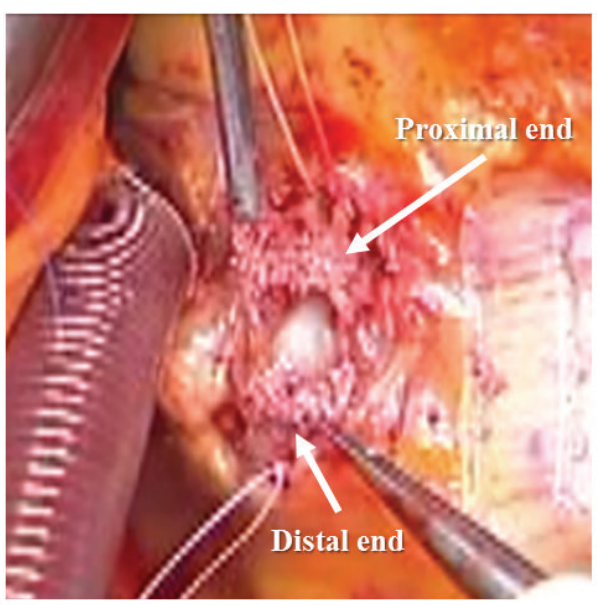

(c)

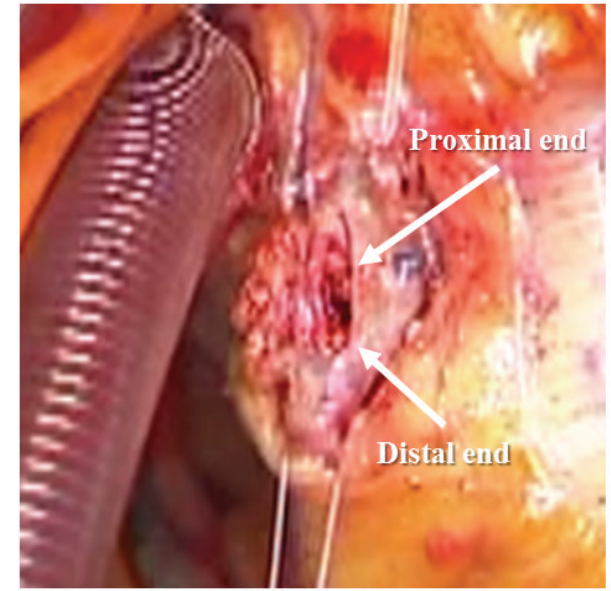

(b)

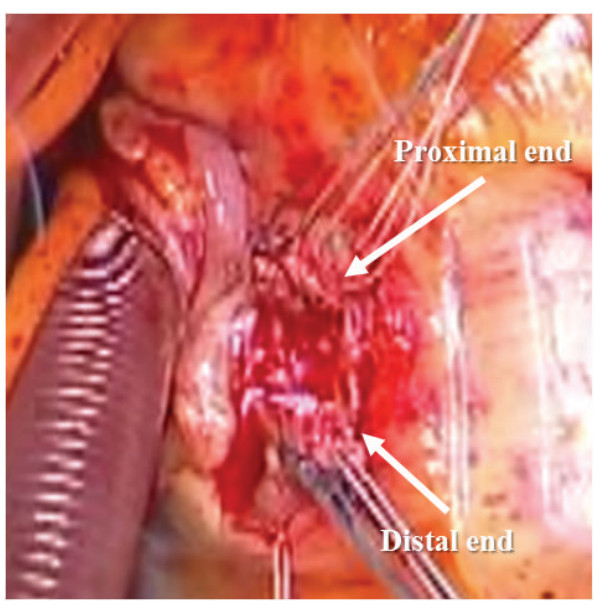

(d)

Figure 5: Intraoperative findings. The pseudoaneurysm is in the visceral adipose tissue (arrow heads) (a) and opened (b). (c) Coronary arterial transection. (d) Proximal and distal transected ends are closed.

University Graduate School of Medicine, for their helpful advice.

\section{References}

[1] S. E. Lee, M. H. Jeong, I. S. Kim et al., "Clinical outcomes and optimal treatment for stent fracture after drug-eluting stent implantation," Journal of Cardiology, vol. 53, no. 3, pp. 422-428, 2009.

[2] G. Nakazawa, A. V. Finn, M. Vorpahl et al., "Incidence and predictors of drug-eluting stent fracture in human coronary artery a pathologic analysis," Journal of the American College of Cardiology, vol. 54, no. 21, pp. 1924-1931, 2009.

[3] Y. Kawai, M. Kitayama, H. Akao, A. Motoyama, T. Tsuchiya, and K. Kajinami, "A case of coronary rupture and pseudoaneurysm formation after fracture of implanted paclitaxel-eluting stents," Cardiovascular Intervention and Therapeutics, pp. 1-7, 2015.

[4] S. Bajaj, R. Parikh, A. Hamdan, and M. Bikkina, "Coveredstent treatment of coronary aneurysm after drug-eluting stent placement," Texas Heart Institute Journal, vol. 37, no. 4, pp. 449454, 2010.
[5] A. Maroo, P. A. Rasmussen, T. J. Masaryk, S. G. Ellis, A. M. Lincoff, and S. Kapadia, "Stent-assisted detachable coil embolization of pseudoaneurysms in the coronary circulation," Catheterization and Cardiovascular Interventions, vol. 68, no. 3, pp. 409-415, 2006.

[6] H. Doi, A. Maehara, G. S. Mintz et al., "Classification and potential mechanisms of intravascular ultrasound patterns of stent fracture," The American Journal of Cardiology, vol. 103, no. 6, pp. 818-823, 2009.

[7] S. Kuramitsu, M. Iwabuchi, T. Haraguchi et al., "Incidence and clinical impact of stent fracture after everolimus-eluting stent implantation," Circulation: Cardiovascular Interventions, vol. 5, no. 5, pp. 663-671, 2012.

[8] R. Virmani, F. Liistro, G. Stankovic et al., "Mechanism of late in-stent restenosis after implantation of a paclitaxel derivateeluting polymer stent system in humans," Circulation, vol. 106, no. 21, pp. 2649-2651, 2002. 


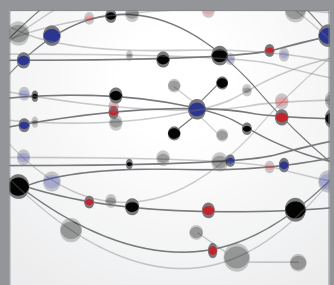

The Scientific World Journal
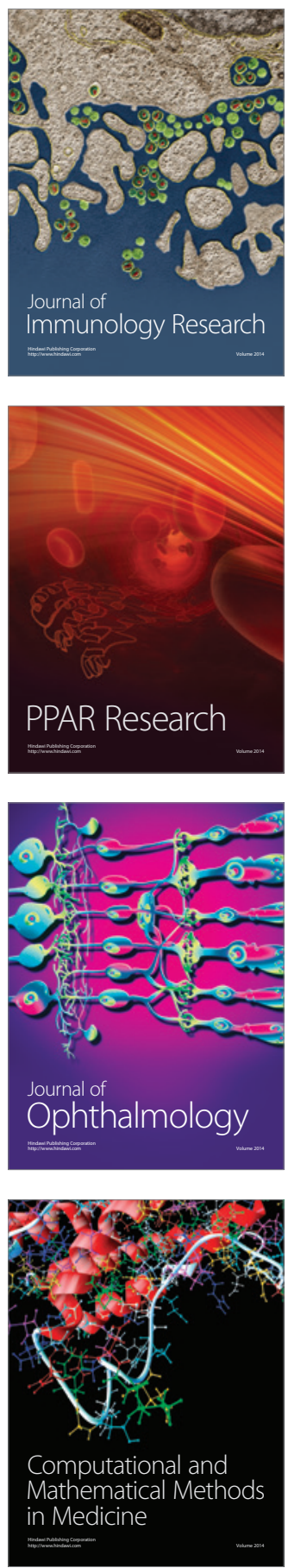

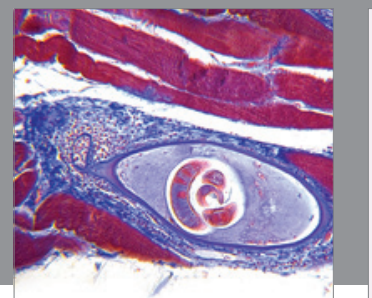

Gastroenterology

Research and Practice
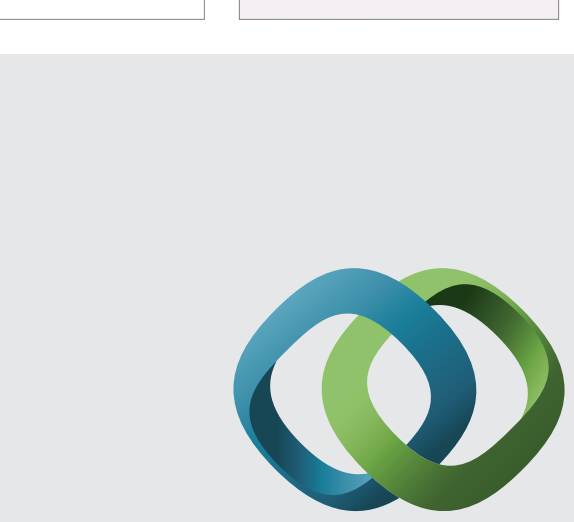

\section{Hindawi}

Submit your manuscripts at

http://www.hindawi.com
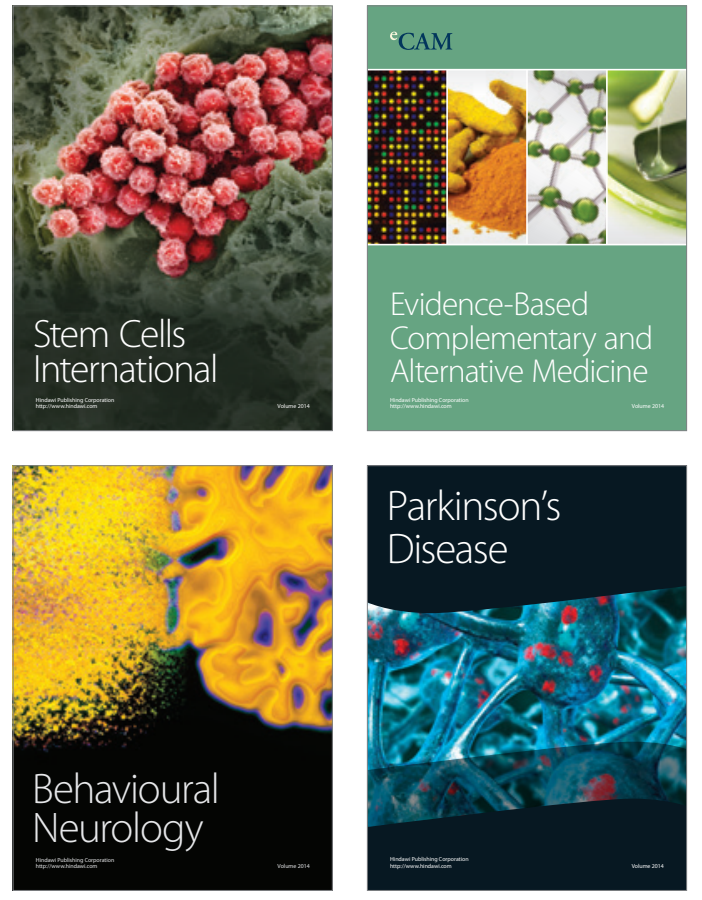
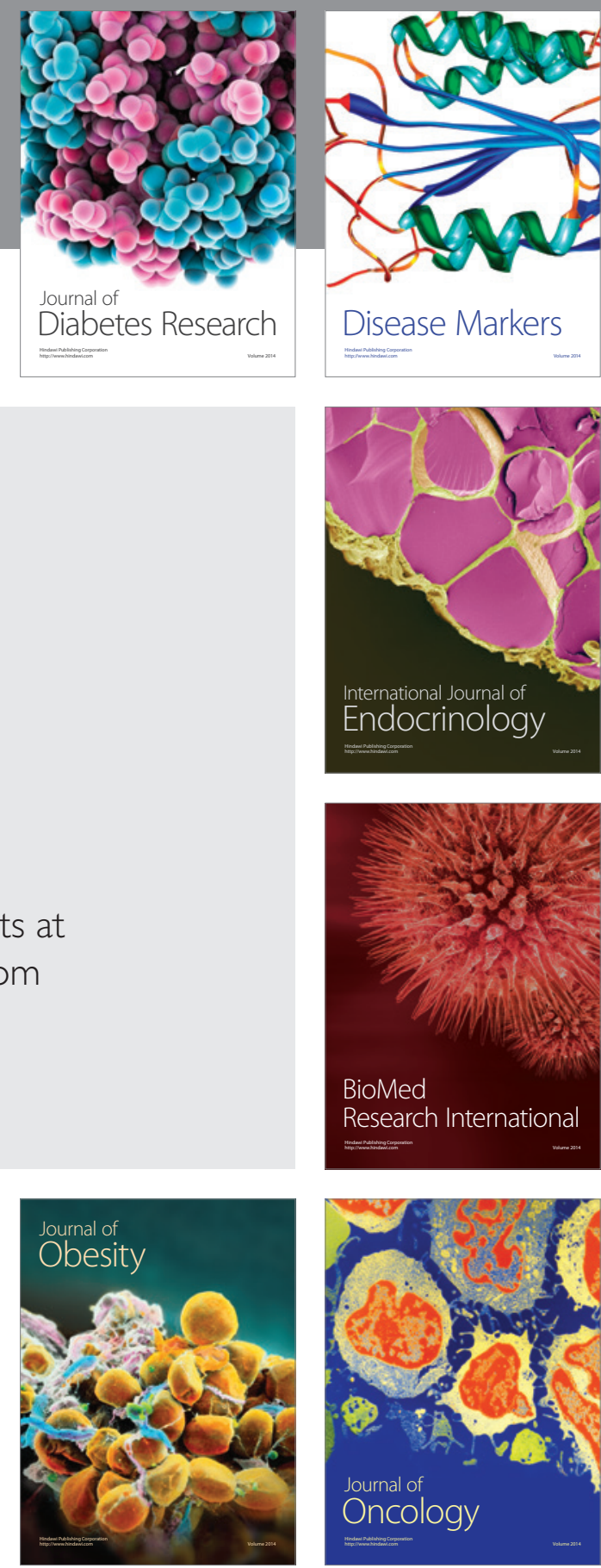

Disease Markers
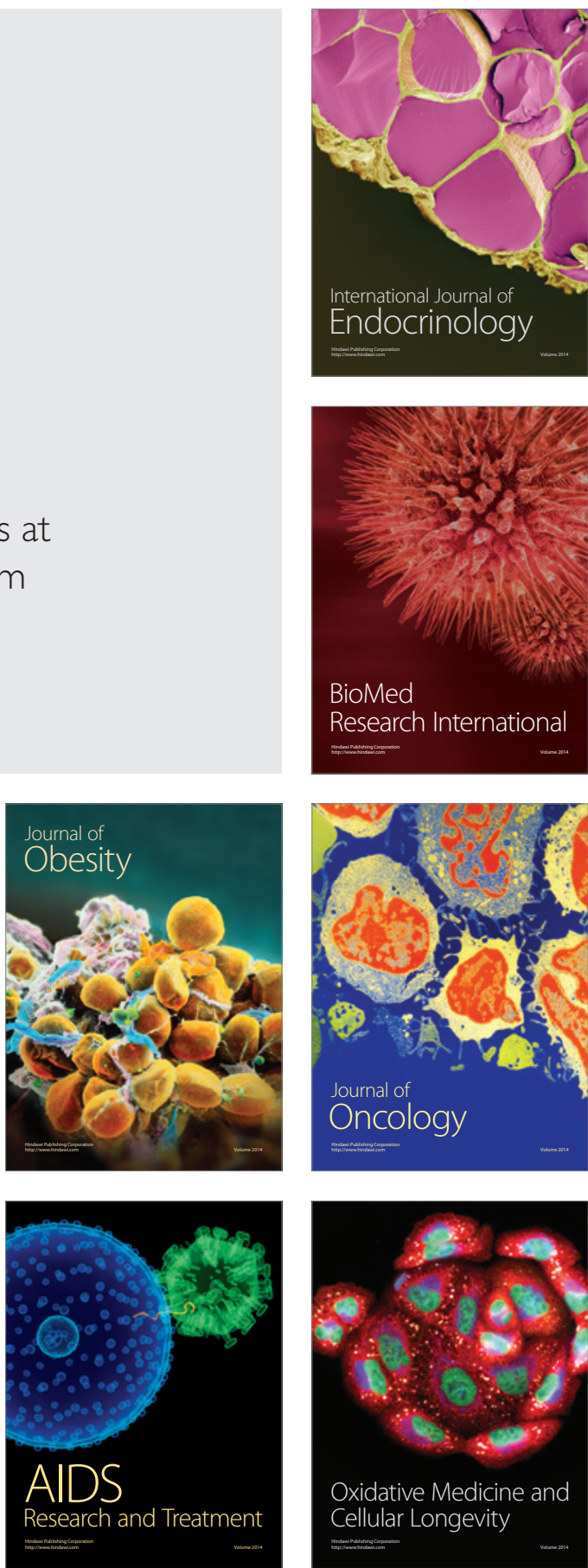